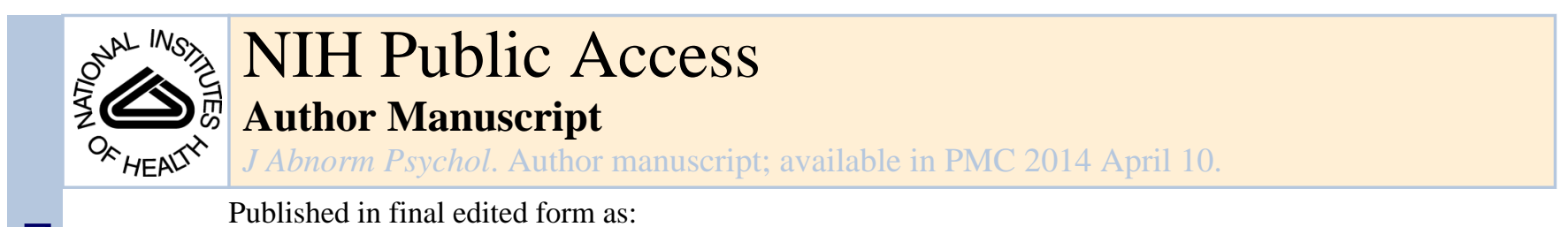

Published in final edited form as:

J Abnorm Psychol. 2012 May ; 121(2): 297-308. doi:10.1037/a0023533.

\title{
Walk on the Bright Side: Physical Activity and Affect in Major Depressive Disorder
}

\author{
Jutta Mata, \\ Stanford University \\ Renee J. Thompson, \\ Stanford University \\ Susanne M. Jaeggi, \\ University of Michigan \\ Martin Buschkuehl, \\ University of Michigan \\ John Jonides, and \\ University of Michigan \\ Ian H. Gotlib \\ Stanford University
}

\section{Abstract}

Although prescribed exercise has been found to improve affect and reduce levels of depression, we do not know how self-initiated everyday physical activity influences levels of positive affect (PA) and negative affect (NA) in depressed persons. Fifty-three individuals diagnosed with Major Depressive Disorder (MDD) and 53 never-depressed controls participated in a seven-day experience sampling study. Participants were prompted randomly eight times per day and answered questions about their physical activity and affective state. Over the week, the two groups of participants did not differ in average level of physical activity. As expected, participants with MDD reported lower average PA and higher average NA than did never-depressed controls. Both participants with MDD and controls reported higher levels of PA at prompts after physical activity than at prompts after inactive periods; moreover, for both groups of participants, PA increased from a prompt after an inactive period to a subsequent prompt at which activity was reported. Depressed participants in particular showed a dose-response effect of physical activity on affect: longer duration and/or higher intensity of physical activity increased their PA significantly more than did short duration and/or lower intensity physical activity. Physical activity did not influence NA in either group. In contrast to previous treatment studies that examined the effects of prescribed structured exercise, this investigation showed that self-initiated physical activity influences PA. These findings also underscore the importance of distinguishing between PA and NA to gain a more comprehensive understanding of the effects of physical activity on affect in MDD.

(C) 2011 American Psychological Association

Correspondence concerning this article should be addressed to Jutta Mata, Department of Psychology, University of Basel, Missionsstrasse62a, 4055 Basel, Switzerland. jutta.mata@unibas.ch.

Jutta Mata, Renee J. Thompson, and Ian H. Gotlib, Department of Psychology, Stanford University; Susanne M. Jaeggi, Martin

Buschkuehl, and John Jonides, Department of Psychology, University of Michigan. 


\section{Keywords}

depression; experience sampling; positive affect; negative affect; exercise

Major Depressive Disorder (MDD) is among the most prevalent of all psychiatric disorders and is associated with enormous personal, familial, and societal costs (Birnbaum et al., 2010). Recent estimates indicate that almost $20 \%$ of the American population, or more than 30 million adults, will experience a clinically significant episode of depression during their lifetime (Kessler \& Wang, 2009). MDD is in large part an affective disorder; indeed, the Diagnostic and Statistical Manual of Mental Disorders IV (DSM-IV) criteria for MDD implicate dysregulated affect as a cardinal symptom of depression. Individuals diagnosed with MDD also use alcohol, nicotine, or illegal drugs (e.g., Merikangas et al., 1996; Sullivan, Fiellin, \& O'Connor, 2005), arguably in an attempt to regulate negative mood (e.g., Abraham \& Fava, 1999). Another, more positive, behavior that is receiving increased attention as a potential "mood regulator" in individuals with MDD (and in the general population) is engaging in physical activity. In this context, several researchers have found that engaging in acute exercise increases positive affect (PA), particularly if PA before exercising was low (see Reed \& Ones, 2006, for a meta-analysis).

Treatment studies using both unselected and clinical samples have found that engaging in prescribed structured exercise significantly reduces levels of depressive symptoms (for meta-analyses see Conn, 2010; Lawlor \& Hopker, 2001; Mead et al., 2009). Indeed, effect sizes comparing intervention and control groups in these meta-analyses were 0.37 in the unselected samples and ranged from 0.82 to 1.1 in the clinical samples, making the effects of exercise comparable to those obtained with cognitive therapy. Although these are large effect sizes, the authors of these meta-analyses noted that methodological difficulties, such as lack of adequate concealment of randomization, lack of blinding, and lack of follow-up assessments beyond the intervention period, make it difficult to determine the true effect size of exercise treatment on reducing depressive symptoms. Moreover, in many of the studies the criterion for "improvement" or "positive outcome" is not clearly specified. For example, several investigations used total scores on the Beck Depression Inventory - II (BDI; Beck, Steer, \& Brown, 1996; Steer, Ball, Ranieri, \& Beck, 1999) to assess the effects of the intervention on depressive symptoms. It is not clear from a single total score, however, which aspects of depressive symptomatology (e.g., positive or negative affect) are changing in response to exercise or physical activity. Finally, the large majority of these studies examined the effects of prescribed or supervised exercise, raising the question of whether these results are generalizable to individuals without access to personal exercise prescription or supervision.

The present study was designed to address a number of these limitations. For example, we assessed both PA and negative affect (NA) to gain a better understanding of the mechanisms by which physical activity might lead to improvement in depression (i.e., by increasing PA vs. decreasing NA). Most of the previous studies examining the relation between exercise and affect have focused on negative psychological states. It is important to note, however, that positive affective states have been found to influence health; for example in unselected samples, positive mood has been found to predict job satisfaction and productivity (George \& Brief, 1992) and improved immune function (Cohen, Doyle, Turner, Alper \& Skoner, 2003). Few studies have examined mood effects in the context of physical activity in participants with MDD. For example, of the 158 studies that Reed and Ones (2006) included in their meta-analysis of the relation between acute exercise and affect, only two explicitly studied participants with MDD (Bartholomew, Morrison, \& Ciccolo, 2005; Bodin \& 
Martinsen, 2004), and these studies had small samples and/or did not include a nondepressed control group.

Given that lower levels of PA before exercise predict a larger change in affect postexercise (cf. Gauvin, Rejeski, \& Reboussin, 2000; Reed \& Ones, 2006), it is possible that the effect of physical activity on affect in depressed individuals is stronger than that in healthy control participants. A consideration of specific psychological and biological factors might also lead to a prediction of a stronger effect of exercise on affect in depressed than in nondepressed individuals. For example, physical activity has been linked to increased self-efficacy and improved self-esteem (Bodin \& Martinsen, 2004), distraction from negative thoughts (Bahrke \& Morgan, 1978; Morgan, 1985), and improved retrieval of positive thoughts (Clark, Milberg, \& Ross, 1983). Because these constructs have been found to be aberrant in depressed individuals (e.g., Gotlib \& Joormann, 2010; Phillips, Hine, \& Thorsteinsson, 2010 for reviews), improving functioning in these domains might have a stronger and more sustained influence on affect in MDD than in control participants. From a more physiological perspective, exercise has been found to stimulate growth of nerve cells and the release of proteins known to improve both the health and survival of nerve cells, such as brain-derived neurotrophic factor (BDNF; e.g., Cotman \& Berchtold, 2002). Importantly, higher levels of physical activity have been shown to be associated with fewer depressive symptoms, particularly in girls with at least one met-allele of the BDNF-gene - which is associated with less BDNF protein release in the brain and higher depressive symptoms compared with girls with the same genotype who engage in little exercise (Mata, Thompson, \& Gotlib, 2010). Thus increased BDNF protein release might also be a mechanism that may explain why people who experience more depressive symptoms could report higher PA and/ or lower NA after being physically active.

In contrast to previous investigations, this study also examines physical activity as it occurs in everyday life instead of supervised exercise bouts. Whereas exercise refers to a subset of physical activity that is planned, structured, repetitive, and purposeful with the goal of achieving improved physical fitness, physical activity refers to all body movement that results in energy expenditure beyond resting expenditure (see Thompson et al., 2003, for a definition of these terms). Differentiating exercise from physical activity, and specifically studying everyday physical activity in individuals with MDD, is particularly important given the difficulty of motivating depressed individuals to engage in and maintain a program of exercise (e.g., Palomo, Beninger, Kostrzewa, \& Archer, 2008).

Previous studies have examined the effects on affect and depressive symptoms of single "bouts" of exercise and of the total amount of exercise per day or per week. Few investigators have examined the possibility that there is a dose-response relation between intensity or duration of physical activity and improvement in mood or depressive symptoms. The results of Dunn, Trivedi, \& O'Neal's (2001) meta-analysis indicate that there may be a dose-response relation between physical activity and improvement in mood, underscoring the importance of examining the effects of duration and intensity of physical activity on affect. In the present study we examine the influence on affect not only of the occurrence of physical activity but also of the intensity and duration of activity.

An ideal method to assess the frequency, duration, and intensity of physical activity and affect in a natural environment is experience sampling, in which participants are prompted by a hand-held device to respond to questions several times per day. This procedure avoids errors associated with long time intervals between measurements (Wadden, Stunkard, \& Smoller, 1986) and with biases of participants when recalling information such as feelings they experienced or activities in which they engaged (Eisenhower, Mathiowetz, \& Morgenstein, 1991). To date, however, few experience sampling studies have assessed both 
physical activity and affect. Gauvin and her colleagues examined the association between affect and engaging in physical activity in healthy middle-aged women using experience sampling (Gauvin, Rejeski, \& Norris, 1996; Gauvin, Rejeski, \& Reboussin, 2000). They found increased PA and decreased NA after engagement in physical activity, particularly if the women were experiencing lower PA before engaging in physical activity. In contrast, Focht, Gauvin, and Rejeski (2004) did not find an association between physical activity and PA or NA in older adults with knee arthritis. Interestingly, Dunton, Atienza, Castro, and King (2009) found that neither PA nor NA predicted subsequent engagement in moderate or strenuous physical activity in healthy older adults. Giacobbi, Hausenblas, and Frye (2005) showed that exercise was associated with increased PA and decreased NA in college students, and Kanning and Schlicht (2010) reported that episodes of daily activity such as walking or gardening increased PA as well as calmness and energetic arousal.

In sum, no study has examined the effect of self-initiated physical activity or exercise on mood over the course of an experience sampling week in clinically depressed individuals. Furthermore, the intensity of physical activity and the possibility of a dose-response relation between physical activity and affect have received little attention. In the present study we used experience sampling to examine the relation between self-initiated physical activity on PA and NA in individuals diagnosed with MDD and healthy controls at various times of day over the course of a week. Because individuals diagnosed with MDD experience diminished pleasure in many activities, in addition to fatigue or loss of energy, we expected that participants with MDD would engage in less physical activity than would healthy controls. We also hypothesized that self-initiated physical activity would be linked with affect. More specifically, for both depressed and control participants we expected that physical activity would be associated concurrently with higher PA and lower NA, and that engaging in physical activity at one prompt would predict higher levels of PA and lower levels of NA at the subsequent prompt. We predicted further that a higher dose of physical activity (i.e., stronger intensity and longer duration) would be associated with a larger increase in PA and a larger decrease in NA. We also expected a larger postactivity change in both PA and NA in individuals diagnosed with MDD than in healthy controls. Finally, because most studies in this area have not examined the role of affect in predicting subsequent engagement in physical activity, we wanted to explore this question as well.

\section{Method}

\section{Participants}

A total of 128 participants were recruited for this study from the communities surrounding the University of Michigan and Stanford University through advertisements posted online (e.g., Craigslist) and at local agencies and businesses in the community (e.g., bulletin boards). Of those, 12 were excluded because of equipment failure, seven because they had a BDI score that was out of range for eligibility (see below) or because they were in partially remitted depressive episodes, and three because they responded to less than one third of the prompts (see Moberly \& Watkins, 2008 for a similar procedure), leaving a final sample of 106 individuals (74 women and 32 men). Fifty-three of these participants were diagnosed with MDD (38 female, 15 male) based on the Structured Clinical Interview for the DSM$I V-R$ (SCID; First, Spitzer, Gibbon, \& Williams, 2001) and 53 were classified as healthy controls (36 female, 17 male). Depressed participants had to meet current $D S M-I V$ criteria for MDD and have a score of 14 or higher on the BDI. They were excluded if they met criteria for Bipolar Disorder, for any psychotic disorders, or for substance dependence within the last six months. Healthy controls could not have had a past or current diagnosis of MDD or any other mental disorder and had to have a BDI score of 9 or lower. All participants were native English speakers. Participants provided informed consent and were 
compensated for their participation in the study, with an extra incentive for responding to more than $90 \%$ of the prompts. The Institutional Review Boards at both the University of Michigan and Stanford University approved the study.

\section{Design and Procedure}

Participants were administered the BDI and the SCID during their first session. Graduate and postbaccalaureate students with extensive training conducted the structured clinical interviews. All interviewers must have demonstrated a solid understanding of psychopathology and diagnostic issues, such as learning $D S M-I V-T R$ criteria, studying the training manual and videotapes, making blind ratings of recorded interviews, and roleplaying interviews. Diagnostic reliability was continually assessed by randomly selecting and rerating recorded interviews. Our team of interviewers has achieved excellent interrater reliability for a major depressive episode $(k=.93)$ and for classifying participants as nonpsychiatric controls $(k=.92$; Levens \& Gotlib, 2010).

If participants were eligible based on SCID criteria, they were invited to a second session in which they completed questionnaires and a battery of tasks assessing working memory (results not reported here). At the end of this session, participants were given a Palm Pilot z22 that prompted them eight times each day between 10 A.M. and 10 P.M. for a total of seven to eight days. Prompts occurred at random times within eight 90-minute windows per day; thus, prompts could occur sometime between a couple of minutes and almost three hours apart. After participants were prompted they had a maximum of three minutes to respond to the questions on the Palm Pilot; otherwise, the Palm Pilot would hibernate until the next prompt. Data were collected using the Experience Sampling Program 4.0 (Barrett \& Feldman Barrett, 2000). Participants returned the palm after the week and were compensated for their participation.

\section{Measures}

In this study we assessed PA and NA by asking participants to rate each of 11 statements, such as "I feel anxious right now," on a four-point scale from "not at all" to "a great deal." The affect words (anxious, sad, disgusted, angry, guilty, ashamed, happy, frustrated, excited, alert, and active) were drawn from various sources, including the Positive Affect Negative Affect Scale (Watson, Clark, \& Tellegen, 1988) and Ekman's basic emotions (e.g., Ekman, Friesen, \& Ellsworth, 1972). To examine the factor structure of these 11 adjectives, we conducted a principal components analysis with varimax rotation. Two components had eigenvalues over Kaiser's criterion of 1 and explained $67.8 \%$ of the total variance. Factor 1, composed of the adjectives assessing NA, explained $45.8 \%$ of the variance, and Factor 2, composed of the adjectives assessing PA, explained $22.0 \%$ of the variance. Reliability for the NA factor was $\alpha=.92$, and for the PA factor was $\alpha=.83$.

To assess physical activity, we asked participants at each prompt "Were you physically active since the last beep?" If they responded affirmatively, we asked them to respond to three follow-up questions: (1) "How long were you active?" with options from 5 to 120 minutes; (2) "What kind of activity did you engage in?" with options of "mild activity (minimal effort; e.g., easy walking, yoga)," "moderate activity (not exhausting; e.g., fast walking, tennis)," and "strenuous activity (heartbeats rapidly; e.g., running, basketball)", and (3) "What activity did you engage in?" where - dependent on their previous answer participants could pick from a list of mild, moderate, or strenuous activities such as badminton, volleyball, swimming etc. Participants were asked to report activities that lasted at least 5 minutes; thus, shorter bouts of activity, such as walking down an office hallway, were not reported. The questions were adapted for the experience sampling methodology from the Godin Leisure-Time Exercise Questionnaire (Godin \& Shepard, 1985). Experience 
sampling questions have been shown to be significantly correlated with objective measures of activity. For example, Grossman, Deuring, Garland, Campbell, and Carlson (2008) reported a correlation of .53 between week-long accelerometry data and the Godin LeisureTime Exercise Questionnaire completed with respect to the accelerometer week. Similarly, Dunton, Whalen, Jamner, Henker, and Floro (2005) found that a measure of heart rate was highly correlated with experience sampling diary reports of various types of exercise intensity in adolescents.

As described above, if participants endorsed being physically active at a prompt, they were asked to respond to three additional questions. To examine whether having to answer additional questions led participants to decrease their reporting of physical activity over the course of the assessment week, we correlated the number of the assessment day ( 1 for the first assessment day, 2 for the second assessment day, etc.) with the number of times participants reported having been physically active each day. There was no significant relation between day of assessment and endorsements of being physically active over the week $(r=-.03, p=.34)$, indicating that the question format did not influence reports of physical activity.

\section{Statistical Model}

Because of the nested structure of our data, in which prompts are nested within days, which in turn are nested within person, we used multilevel modeling for our analyses (program version HLM 6.08). Hierarchical linear modeling (HLM; also referred to as multilevel random coefficient models) is an extension of the regression approach. HLM analyzes data separately at the level of prompts and at the level of participants, allowing us to test relations among both within- and between-level data without violating standard assumptions of independence. Importantly, HLM accommodates missing data (not all participants responded to all prompts) and unequal time intervals between prompts (Nezlek, 2001). We used a two-level model with prompts nested within participants. ${ }^{1}$

\section{Results}

The Results section is divided into three parts. In the first part, after describing the demographic and clinical characteristics of the sample, we present data concerning the number of prompts to which participants responded and their levels of affect and activity over the experience sampling week. In the second part, we report concurrent associations between physical activity and affect, examining whether affect on prompts or days with physical activity differed from affect on no-activity prompts or days. In the third section, we describe time-lag effects, that is, changes in affect from one prompt or from one day to the next as a function of engaging in physical activity. We also examine the trajectory of affect before and after a bout of physical activity. In all of these analyses we compare the functioning of depressed and never-depressed control participants.

\section{Demographic and Clinical Characteristics of Participants}

Demographic and clinical characteristics of the participants are presented in Table 1. Participants recruited from the Stanford and Michigan communities differed with respect to age [Michigan: 24.2 years; Stanford: 29.7 years, $t(104)=4.69, p<.001$ ], education level (more Stanford participants had a Bachelor's or Master's degree, whereas Michigan participants had "some college education", $\left.\chi^{2}(3)=9.67, p=.02\right)$, and gender distribution (more males were recruited in Michigan, $\chi^{2}(1)=11.78, p<.001$ ). Importantly, the two sites

\footnotetext{
${ }^{1}$ We could not run a three-level model because there were a substantial number of days without reported physical activity (44\%), which would exclude these days from the analyses.
} 
did not differ in the number of participants recruited and classified as MDD or control, in BDI scores, or in ethnicity (all $p>.05$ ). Given that the two sites did not differ with respect to these primary variables of interest, Table 1 presents combined data from the two sites, separately by diagnostic group. As expected, participants with MDD reported higher BDI scores than did controls. In addition, participants with MDD were, on average, about three years older than controls; no other group differences were significant. Importantly, as can be seen in Table 1, the depressed and control participants did not differ in the number of prompts to which they responded.

\section{Do Depressed and Control Participants Differ in Affect and Physical Activity?}

Consistent with findings of previous studies (e.g., Jolly, Dyck, Kramer, \& Wherry, 1994;

Watson, Clark, Weber, \& Assenheimer, 1995), depressed participants reported significantly lower PA and significantly higher NA over the experience sampling week than did control participants (see Table 2). Because the physical activity data were not normally distributed (except for average duration), we report medians and use Mann-Whitney tests to analyze these data. Notably, in contrast to our hypotheses, there were no differences between depressed and control participants in frequency, intensity, or duration of physical activity over the week. There was one exception: never-depressed control participants reported engaging in strenuous activity more often during the one-week assessment period than did depressed participants.

\section{Are Physical Activity and Affect Associated Differentially in Depressed and Control Participants? Concurrent Associations on the Prompt Level}

To examine whether depressed and control participants differ in the "affective benefit" they derive from physical activity, we compared affect at prompts at which participants reported having engaged in physical activity with affect at prompts at which they reported having engaged in no physical activity. We tested two models that differed with respect to whether prompt-level PA or prompt-level NA was the dependent variable. At the level of prompts, our primary independent variable was a dummy variable, reflecting whether participants were physically active (coded 1) or were not physically active (coded 0 ) at the time of that prompt. We also included linear and quadratic effects of time of day, operationalized as the number of minutes since the first prompt of the day (centered across all participants). At the level of participants, diagnostic group-MDD (coded 0) versus control (coded 1) —was our sole predictor. ${ }^{2}$ The equations for the full HLM model are presented below, with ${ }_{i}$ representing prompts and ${ }_{j}$ representing participants.

Model at the Level of prompts:

$$
\begin{aligned}
& \text { Affect } \left._{\mathrm{ij}} \text { (either positive or negative }\right) \\
& \quad=\beta_{0 \mathrm{j}}+\beta_{1 \mathrm{j}}(\text { physically active or not })+\beta_{2 \mathrm{j}}(\text { time of day })+\beta_{3 \mathrm{j}}(\text { quadratic time of day })+r_{\mathrm{ij}} .
\end{aligned}
$$

Model at the Level of participants:

$$
\begin{gathered}
\left.\beta_{0 \mathrm{j}}=\gamma_{00}+\gamma_{01} \text { (diagnostic group }\right)+u_{0 \mathrm{j}} . \\
\beta_{1 \mathrm{j}}=\gamma_{10}+\gamma_{11} \text { (diagnostic group) } \\
\beta_{2 \mathrm{j}}=\gamma_{20}+\gamma_{21} \text { (diagnostic group). } \\
\beta_{3 \mathrm{j}}=\gamma_{30}+\gamma_{31} \text { (diagnostic group). }
\end{gathered}
$$

\footnotetext{
${ }^{2}$ In additional HLM models we tested the effects of gender and the interaction between diagnostic group and gender; none of these predictors was significant.
} 
Affect $_{\mathrm{ij}}$ represents affect for person ${ }_{j}$ on prompt $_{i}, \beta_{0 \mathrm{j}}$ represents each participant's mean of affect across all prompts without physical activity, $\beta_{1 \mathrm{j}}$ is the difference in the average level of affect on a prompt with activity reported versus no activity reported, $\beta_{2 \mathrm{j}}$ represents the linear time of day effects in affect, and $\beta_{3 j}$ quadratic time of day effects in affect; $r_{i j}$ represents the within-person residuals. $\gamma_{00}$ is the average affect on prompts on which no physical activity was reported for individuals with MDD, and $\gamma_{01}$ reflects the difference on prompts without physical activity between the two participant groups. $\gamma_{10}$ is the difference between affect on prompts without physical activity versus with physical activity for depressed participants, and $\gamma_{11}$ represents the difference in affect across prompts with versus without physical activity between depressed and control participants.

Positive affect-On prompts without physical activity, the average PA for MDD participants was $\gamma_{00}=1.64, S E=0.06, t(103)=27.39, p<.001$; PA for control participants was significantly higher by one half of a scale point, $\gamma_{01}=0.48, S E=0.08, t(103)=5.67, p$ $<.001$. On prompts with physical activity, PA in both depressed and control participants was significantly higher than it was on prompts for which subjects reported being inactive. In depressed participants, this increase was one third of a scale point, $\gamma_{10}=0.32, S E=0.03$, $t(4272)=10.34, p<.001$. In control participants, the increase in PA was similar in magnitude to that in MDD participants, $\gamma_{11}=-0.05, S E=0.04, t(4272)=-1.17, p=.24$. Thus, the interaction between diagnostic group (MDD vs. control) and being physically active or not on PA is not significant in this model. Because results from HLM models are presented relative to each other, the value of $\gamma_{11}=-0.05$ means that the increase in PA on prompts with activity in control participants was only -0.05 scale point lower than that of the participants with MDD $\left(\gamma_{10}=0.32\right)$. Thus, both depressed and control participants reported significantly (and nondifferentially) higher PA on prompts at which physical activity was reported than on prompts without physical activity (see Figure 1).

Negative affect-For participants with MDD, the average NA on prompts without physical activity was $\gamma_{00}=1.88, S E=0.05, t(103)=34.28, p<.001$, about three-quarters of a scale point higher than that of control participants $\gamma_{01}=-0.74, S E=0.08, t(103)=9.62, p$ $<.001$. NA was not significantly different on prompts with physical activity than on prompts without activity for either MDD $\left(\gamma_{10}=-0.03, S E=0.03, t(4272)=-1.10, p=.27\right)$ or control participants $\left(\gamma_{11}=0.02, S E=0.03, t(4272)=0.65, p=.52\right)$, indicating that there was no change in NA between prompts as a function of physical activity for either participant group (see Figure 1). ${ }^{3}$

Intensity and duration of activity and affect-Finally, we examined whether there are characteristics of physical activity that are particularly associated with levels of PA or NA. Specifically, we assessed the effect of dose of physical activity on affect by combining duration of the activity with its intensity into "METminutes" (MET = metabolic equivalent of task, expressing the energy cost of physical activity). METminutes are the duration of an activity multiplied by its MET. For this study, we used the standards defined in the International Physical Activity Questionnaire (Craig et al., 2003), that is, 3.3 METminutes for mild activity, 4.0 for moderate activity, and 8.0 for strenuous activity. For example, 10 minutes of strenuous activity are equivalent to $10 \times 8=80$ METminutes. The HLM models used to analyze the influence of METminutes on affect are identical to the equations above.

\footnotetext{
${ }^{3}$ These results for PA and NA hold when controlling for the influence of time-of-day effects by adding minutes since the first prompt of the day as predictors at the within-person level. We found significant linear and quadratic time-of-day effects for PA showing that PA is lower in the evening and an inverted U-shaped quadratic trend reflecting higher PA in the middle of the day than in the morning or evening [linear: $t(4285)=-2.59, p<.001$; quadratic: $t(4285)=-3.88, p<.001]$. There were no differences between MDD and control participants in time-of-day effects for PA, nor were there significant linear or quadratic time-of-day effects for NA or group differences for these variables.
} 
We simply replaced being physically active or not as an independent variable at the prompt level with the predictor MET-minutes. We found that overall the more METminutes of physical activity in which depressed participants engage the higher their PA $\left(\gamma_{10}=0.00057\right.$, $S E=0.00022, t(905)=2.58, p=.01)$. The impact of METminutes on PA is much weaker in control participants $\left(\gamma_{11}=-0.00043, S E=0.00033, t(905)=-1.31, p=.19\right)$, but the interaction between METminutes and diagnostic group is not significant. There was no relation between METminutes and NA for either group. Notably, although the difference between depressed and control participants is not significant, increase in PA per METminute is more than twice as high as it is in the control group.

\section{Concurrent Associations Between Physical Activity and Affect on the Day Level}

To test whether being physically active was associated with affect at a broader level than a single prompt, we conducted similar analyses on a day level by testing the following model:

Model at the Level of days:

Affect $_{\mathrm{ij}}($ either positive or negative $)=\beta_{0 \mathrm{j}}+\beta_{1 \mathrm{j}}$ (physically active or not $)+\beta_{2 \mathrm{j}}($ measurement day $)+r_{\mathrm{ij}}$.

Model at the Level of participants:

$$
\begin{gathered}
\left.\beta_{0 \mathrm{j}}=\gamma_{00}+\gamma_{01} \text { (diagnostic group }\right)+u_{0 \mathrm{j}} . \\
\left.\beta_{1 \mathrm{j}}=\gamma_{10}+\gamma_{11} \text { (diagnostic group }\right) . \\
\beta_{2 \mathrm{j}}=\gamma_{20}+\gamma_{21} \text { (diagnostic group). }
\end{gathered}
$$

Positive affect-The daily average of PA in depressed participants on an inactive day was $\gamma_{00}=1.58, S E=0.06, t(103)=28.43, p<.001$, and increased by one fifth of a scale point on days with physical activity $\left(\gamma_{10}=0.17, S E=0.03, t(829)=5.09, p<.001\right)$. In control participants, PA on days with physical activity was one half of a scale point higher than that of depressed individuals, $\gamma_{01}=0.54, S E=0.09, t(103)=5.95, p<.001$. Thus, depressed participants have higher levels of PA on active than on inactive days. The increase in PA from inactive to active days was lower for controls than for depressed participants, $\gamma_{11}=$ $-0.11, S E=0.05, t(829)=-1.91, p=.06$, showing a nearly significant interaction between active versus inactive days and diagnostic group, suggesting that the increase in PA from inactive to active days is larger in individuals with MDD than in control participants.

Negative affect-On inactive days NA in individuals with MDD was $\gamma_{00}=1.58, S E=$ $0.06, t(103)=28.43, p<.001$, and was not significantly different on active days, $\gamma_{10}=$ $-0.02, S E=0.04, t(829)=-0.75, p=.45$. For control participants, NA on inactive days was significantly lower than that of depressed individuals, $\gamma_{01}=-0.75, S E=0.08, t(103)=$ $-9.64, p<.001$, but also was not significantly different on active days, $\gamma_{11}=0.02, S E=$ $0.04, t(829)=0.60, p=.55$. Thus, there was no significant change in NA from active to inactive days for either group of participants.

Intensity and duration of activity and affect-The results described above on the influence of METminutes on affect reported at the prompt level were replicated in direction, magnitude, and significance on the day level. Again, we found that overall the more METminutes of physical activity in which depressed participants engage, the higher their PA over the day $\left[\gamma_{10}=0.00026, S E=0.00010, t(461)=2.56, p=.01\right]$. The same relation is true for control participants [ $\gamma_{11}=-0.00009, S E=0.00014, t(461)=-0.59, p=.56$ ]; albeit the impact of METminutes on PA is weaker in the control group, the interaction between 
METminutes and diagnostic group is not significant. There was no relation between METminutes of physical activity and NA in either depressed or control participants.

\section{Effects of Physical Activity on Subsequent Affect at the Prompt Level}

In this section we report changes in PA and NA from one prompt to the next as a function of whether physical activity was reported at the second prompt. (Note that it is most likely that physical activity occurred during the interval between two prompts and was reported at the second prompt). If physical activity increases PA, we should see an increase in PA from a prompt without activity to the next prompt at which activity is reported, but no such increase in PA between two consecutive prompts at which no activity occurred. A positive difference score in PA or NA means that affect increased from prompt to prompt $t_{t+1}$ if physical activity occurred at $t+1$. To control for potential confounding effects of previous physical activity, we excluded subsequent prompts if either physical activity occurred at ${ }_{\mathrm{t}}$ but not at $\mathrm{t}+1$ or at which physical activity occurred at both prompts.

We compared prompts at which no physical activity is reported at both ${ }_{t}$ and $t+1$ with prompts at which physical activity is reported at $t_{t+1}$ but not at ${ }_{t}$. We excluded prompts $s_{t+1}$ that occurred on the following day. To analyze whether affect changes from prompt to prompt $_{\mathrm{t}+1}$ if physical activity is reported at $\mathrm{t}+1$, we tested the following model:

Model at the Level of prompts:

$$
\begin{aligned}
& \text { Affect }_{\mathrm{ij}(\mathrm{t}+1)}(\text { either positive or negative }) \\
& \quad-\text { affect }_{\mathrm{ij}(\mathrm{t})}(\text { either positive or negative }) \\
& \quad=\beta_{0 \mathrm{j}}+\beta_{1 \mathrm{j}}\left(\text { physically active or not } \mathrm{t}_{\mathrm{t}+1}\right) \\
& \quad+\beta_{2 \mathrm{j}}(\text { time of day })+\beta_{3 \mathrm{j}}(\text { quadratic time of day })+r_{\mathrm{ij}} .
\end{aligned}
$$

Model at the Level of participants:

$$
\begin{gathered}
\left.\beta_{0 \mathrm{j}}=\gamma_{00}+\gamma_{01} \text { (diagnostic group }\right)+u_{0 \mathrm{j}} . \\
\beta_{1 \mathrm{j}}=\gamma_{10}+\gamma_{11} \text { (diagnostic group). } \\
\beta_{2 \mathrm{j}}=\gamma_{20}+\gamma_{21} \text { (diagnostic group). } \\
\beta_{3 \mathrm{j}}=\gamma_{30}+\gamma_{31} \text { (diagnostic group). }
\end{gathered}
$$

Positive affect-As expected, for both depressed and control participants the difference in PA between prompt $t_{t+1}$ and prompt $_{t}$ when no physical activity was reported at either prompt did not differ significantly from zero [depressed participants: $\gamma_{00}=0.05, S E=0.03, t(103)=$ $1.81, p=.07$; control participants: $\left.\gamma_{01}=0.01, S E=0.04, t(103)=0.17, p=.87\right]$. When physical activity was reported at prompt $_{\mathrm{t}+1}$, PA increased in both groups: in depressed participants by one-quarter of a scale point, $\gamma_{10}=0.28, S E=0.05, t(2717)=5.71, p<.001$, and in control participants by a similar magnitude, $\gamma_{10}=-0.07, S E=0.07, t(2717)=-0.97$, $p=.33$. In sum, for both depressed and control Participants PA increased from prompts at which no physical activity was reported to prompts at which they reported engaging in physical activity. The interaction between diagnostic group and being physically active or not at the second prompt was not significant (see Figure 2).

Negative affect-There was no difference in NA either between two consecutive prompts without physical activity or between an inactive and a subsequent active prompt for either participant group (all $p>.05$ ). Again, these results hold even when controlling for the influence of linear or quadratic time effects on affect over the day. 


\section{Effects of Physical Activity on Subsequent Affect at the Day Level}

Positive affect-Next, we tested time-lag effects at a day level to examine whether affect changed over two consecutive days without physical activity compared with a day without physical activity followed by a day with physical activity. We found that for both depressed and control participants, the difference in PA between two consecutive days without physical activity is small, $\gamma_{00}=-0.078, S E=0.038, t(92)^{4}=-2.05, p=.04$, and $\gamma_{01}=0.03$, $S E=0.05, t(92)=0.49, p=.62$, respectively. In contrast, there was a significant increase in PA from a day without physical activity to a day with physical activity in both MDD [ $\gamma_{10}=$ $0.22, S E=0.059 ; t(302)=3.80, p<.001]$ and control participants $\left[\gamma_{11}=-0.10, S E=0.09\right.$, $t(302)=-1.14, p=.25]$. Again, the nonsignificant $\gamma_{11}$ value indicates that there was no interaction between being physically active or not and diagnostic group. Thus, there was no difference in the magnitude of the increase in PA between depressed and control participants; both groups had a significant increase in PA from a nonactive day to an active day.

Negative affect-Again, there was no difference in NA between two consecutive days without physical activity compared with a day with physical activity that was followed by a day without physical activity for either depressed or control participants (all $p>.05){ }^{5}$

\section{Trajectories of Affect Over the Day Before and After Being Physically Active}

We next examined the trajectories of affect before and after being physically active. To avoid potential confounds, we confined these analyses to the first bout of physical activity that was reported at that day. That is, for each day we examined in separate analyses the trajectory of affect before and after the first bout of physical activity. We examined only the first bout of physical activity to control for the possible reciprocal effects of affect and multiple bouts of physical activity. For these analyses we used minutes before the first physical activity prompt and minutes after the physical activity prompt, with the prompt at which physical activity was reported being 0 . For example, if the prompt before the first prompt at which physical activity was reported happened 89 minutes before, we coded it as "-89;" if the prompt after the first prompt at which physical activity was reported happened after 95 minutes, we coded it as "95." In addition to this linear time variable, we also tested possible quadratic time effects of affect before and after the first physically active prompt.

Model at the level of prompts for trajectory of affect before physical activity:

$$
\begin{aligned}
& \text { Affect }_{\mathrm{ij}} \text { (either positive or negative) } \\
& \left.\quad=\beta_{0 \mathrm{j}}+\beta_{1 \mathrm{j}} \text { (time before physical activity in minutes_linear }\right) \\
& \quad+\beta_{2 \mathrm{j}}(\text { time before physical activity in minutes } \text { quadratic })_{-}+r_{\mathrm{ij}} .
\end{aligned}
$$

Model at the Level of participants:

\footnotetext{
${ }^{4}$ Degrees of freedom in this analysis are lower because 11 participants did not have either two consecutive days of inactivity or a day of inactivity followed by a day on which physical activity occurred.

5 To exclude the possibility that increased PA reported at prompts at which physical activity occurred is attributable to the pleasantness of engaging in an activity compared with not being active, we reran all concurrent prompt- and day-level analyses that compare PA at prompts with physical activity with PA at prompts without physical activity. In these additional analyses we controlled for whether participants rated the physical activity as exciting, neutral, or boring by adding this item as a predictor in the model. The original results replicated on both the prompt and the day level, indicating that PA is increased on prompts and days with physical activity regardless of the rated pleasantness/arousal of the activity in which participants were engaged.
} 


$$
\begin{gathered}
\left.\beta_{0 \mathrm{j}}=\gamma_{00}+\gamma_{01} \text { (diagnostic group }\right)+u_{0 \mathrm{j}} . \\
\left.\beta_{1 \mathrm{j}}=\gamma_{10}+\gamma_{11} \text { (diagnostic group }\right) . \\
\left.\beta_{2 \mathrm{j}}=\gamma_{20}+\gamma_{21} \text { (diagnostic group }\right) .
\end{gathered}
$$

The intercept $\left(\gamma_{00}\right)$ shows affect at the prompt at which physical activity was reported. If the coefficient $\gamma_{10}$ is positive, then there is a linear increase in affect the closer in time the participant is to the prompt at which physical activity occurs. A positive coefficient $\gamma_{20}$ represents a positive quadratic effect of time of affect before the first physical activity bout of the day The coefficients $\gamma_{01}, \gamma_{11}, \gamma_{21}$ show whether there is an interaction between diagnostic group and affect at the prompt at which physical activity is reported, an interaction between diagnostic group and linear trajectory of time with respect to affect before the physically active prompt, and an interaction between diagnostic group and quadratic trajectory of time with respect to affect before the physically active prompt, respectively.

Trajectory of affect before physical activity-At the first prompt of the day at which physical activity occurred, we found that PA was significantly different from zero for the MDD group $\left(\gamma_{00}=1.96, S E=0.07, t(102)=28.21, p<.001\right)$. PA for control participants at the first physically active prompt was one third of a scale point higher and significantly different than that of the MDD group, $\gamma_{01}=0.38, S E=0.10, t(102)=3.86, p<.001$. The less time remaining until the first physical activity bout of the day occurs, the higher the level of PA in the MDD group. In depressed participants, this increase was 0.002 scale points for each minute that the participant got closer to reporting being physically active, $\gamma_{10}$ $=0.002, S E=0.0003, t(1160)=5.28, p<.001$. For control participants, the increase in PA was similar in magnitude to that of participants with MDD, $\gamma_{11}=0.0004, S E=0.0005$, $t(1160)=0.71, p=.48$. The quadratic time trajectory of PA before the first bout of physical activity of the day was also significant in participants with MDD, $\gamma_{20}=0.000002, S E=$ $0.000001, t(1160)=2.61, p=.009$, and, again, not significantly different from the quadratic trajectory of affect in control participants, $\gamma_{21}=0.000001, S E=0.000001, t(1160)=1.16, p$ $=.25$. Thus, increases in PA as participants get closer to the first bout of physical activity of the day are of similar magnitude in both diagnostic groups. As in the previous analyses, we did not find either a positive or a negative linear or quadratic trajectory of NA before the first physically active bout of the day for either depressed or control participants (all $p>$. $05)$.

Trajectory of affect after physical activity-Next, we tested the trajectory of affect for the time after the first bout of the day physical activity occurred. The statistical model is analogous to the model presented in Equations 7 and 8 with "time after the first bout of physical activity of the day occurred" instead of time before the first bout of physical activity occurred. For the trajectory of PA after the first prompt of the day at which physical activity occurred, we found that the more time that has passed since the first physical activity bout of the day, the lower the level of PA in the MDD group. In depressed participants, this linear decrease was 0.001 scale points for each minute that had passed since the participant reported being physically active, $\gamma_{10}=-0.001, S E=0.0004, t(1152)=$ $-2.63, p=.009$. For control participants, the decrease in PA was similar in magnitude to that of depressed participants, $\gamma_{11}=-0.0003, S E=0.0006, t(1152)=-0.48, p=.63$. Thus, the effect of the interaction between diagnostic group and linear decrease in PA after the first bout of physical activity is not significant in this model. Furthermore, the quadratic time trajectory of PA after the first bout of physical activity of the day was not significant in depressed participants, $\gamma_{20}=0.000001, S E=0.000001, t(1152)=1.22, p=.22$, and, again, not significantly different from the quadratic time trajectory in control participants, $\gamma_{21}=$ 
0.000001, $S E=0.000001, t(1152)=0.92, p=.36$. Again, we did not find either a positive or a negative linear or quadratic trajectory of NA after the first physically active bout of the day for either depressed or control participants (all $p>.05$ ). In sum, PA decreases over time after the first bout of physical activity, and depressed and control participants do not differ in the trajectories of PA and NA after being physically active.

\section{Discussion}

In this study we examined the association between self-initiated physical activity and PA and NA in individuals diagnosed with MDD compared with healthy controls. Contrary to our hypothesis, the depressed and control participants did not differ in their overall level of physical activity over the experience sampling week. As we predicted, we found that in both Groups PA was higher at prompts at which physical activity had occurred than at prompts without physical activity. In fact, compared with nondepressed controls, depressed participants reported higher average PA on days with physical activity than on days without physical activity, supporting our hypothesis of a larger effect of physical activity on PA in depressed than in control participants at a day level. Consistent with our hypothesis, we also found a dose-response effect, in which increased intensity and duration of physical exercise was associated with a greater increase in PA; this dose-response effect was more pronounced in individuals with MDD than it was in healthy controls. In addition, we found that PA increased from one occasion to the next if participants were physically active during that interval, suggesting that physical activity precedes increased PA. Finally, we found that PA increases over time before, and decreases after, the first reported bout of physical activity of the day in both depressed and control participants.

Interestingly, and contrary to our predictions, there was no relation between physical activity and NA in either the depressed or the control participants. This finding stands in contrast to results of other experience sampling studies conducted with unselected samples of participants (e.g., Gauvin, Rejeski, \& Norris, 1996; Gauvin, Rejeski, \& Reboussin, 2000; Giacobbi et al., 2005). One notable difference between these investigations and the present study is that our comparison group consisted of individuals with no current or past Axis I psychiatric disorders. The unselected samples in previous studies were likely more heterogeneous with respect to the presence of psychiatric disorders than were our healthy controls and, consequently, likely experienced higher mean levels and greater variability in NA than did our control participants. In this context we should also note that although, as expected, NA was higher in the depressed participants than it was in controls, we did not find an association between engaging in physical activity and NA in this group either, tempering the viability of this explanation. The association between physical exercise and $\mathrm{PA}$, but not NA, in MDD adds to a growing literature documenting the importance of PA in depression, perhaps to an even greater extent than NA (cf. Watson et al., 1995). For example, Canli et al. (2004) and Pizzagalli, Iosifescu, Hallett, Ratner, and Fava (2008) found depressed individuals to be less responsive than were nondepressed controls to positive stimuli. Deldin, Deveney, Kim, Casas, and Best (2001) found lower N200 event-related brain potential (ERPs) in response to positive faces in depressed than in nondepressed individuals, potentially reflecting decreased resource allocation to positive stimuli. The dissociation of PA and NA in the depressed participants in this study underscores the importance of investigators continuing to examine differences between the relations of PA and NA with depression.

Contrary to our predictions, we did not observe a difference between individuals with MDD and healthy controls in general activity level. It is possible that those depressed participants who were motivated to participate in a research study have a relatively active daily life. We did, however, observe a lower level of strenuous activity in participants with MDD than in 
controls, suggesting that depressed individuals engaged in less planned exercise (e.g., strenuous running), which is consistent with their experience of fatigue and loss of energy. We found a dose-response relation between physical activity and PA in the present study such that more METminutes were associated with higher PA. This finding is consistent with Dunn, Trivedi, \& O'Neal's (2001) meta-analysis that suggested such a dose-response relation. Other studies have found that physical activity of all intensities (from light to vigorous) is associated with lower levels of depressive symptoms (Hamer, Molloy, de Oliveira, \& Demakakos, 2009; Lindwall, Rennemark, Halling, Berglund, \& Hassmen, 2007), and that lower-intensity activity leads to a stronger increase in PA in nondepressed adults (Conn, 2010) and in unselected elderly adults (Arent, Landers, \& Etnier, 2000). One explanation for these discrepant findings is that, unlike in most intervention studies, participants in our study self-selected their activity intensity. Ekkekakis' (2003) dual-mode theory suggests that exercise affects mood and depressive symptoms jointly by top-down cognitive factors (e.g., exercise self-efficacy) and bottom-up interoceptive afferents (e.g., muscular and respiratory cues). The relative importance of these two factors is assumed to shift as a function of exercise intensity: cognitive factors are dominant at low to moderate exercise intensities (improving affect) and interoceptive cues are more salient as exercise approaches an individual's functional limits (being associated more with exhaustion). If participants feel efficacious and comfortable at their self-selected intensity level, this might explain why we found a dose-response relation in our sample when this relation was not found in samples in which exercise intensity is prescribed and participants' activity may be closer to a level of exhaustion. In this context, it is noteworthy that Knapen et al. (2009) found that self-selected exercise intensity, and not prescribed exercise intensity, increased positive well-being in depressed individuals. Thus, it is important in future research to continue to assess the relation between activity intensity and levels of affect and depressive symptomatology.

The finding that only depressed participants had higher PA on active than on nonactive days is consistent with the observation that coefficients of increase in PA attributable to activity were generally larger in the MDD group. One explanation for this finding is that despite the observation that both the depressed and the control participants benefit from physical activity, the control participants might experience more positive events over the day that enhance their PA over and above the increase derived from being physically active. As a related point, physical activity might counteract the negative cognitive biases that have been found to characterize depressed individuals (Gotlib, Krasnoperova, Yue, \& Joormann, 2004; Joormann, Teachman, \& Gotlib, 2009) and thereby increase their level of PA. Finally, other exercise-induced changes in psychological (e.g., self-efficacy, self-esteem) or biological (e.g., stimulation of BDNF) mechanisms might also contribute to the generally stronger association between being physically active and PA in the depressed participants. Future research is required to examine these possibilities more systematically and explicitly.

In this manuscript we also explored the trajectory of affect over the day before and after the first bout of reported physical activity. Although the vast majority of studies and metaanalyses have examined the effect of physical activity on affect, few studies have examined whether higher PA precedes becoming physically active. Dunton and colleagues (2009) did not find that level of affect predicts physical activity. In contrast, our results show that PA increases over time before the first bout of physical activity and slowly decreases after the prompt at which physical activity is reported. The pattern of findings suggests that PA is highest at the time of physical activity. Nevertheless, given that these are observational data, we cannot determine whether the increase in PA leads participants to become physically active or whether the anticipation of physical activity increases PA. 
Perhaps because of potential difficulty in participant compliance and the need to use more complex analytic strategies (e.g., Ebner-Priemer, Eid, Kleindienst, Stabenow, \& Trull, 2009), experience sampling methods have not been used widely to date in research examining psychopathology. Investigators utilizing these methods, however, have reported findings that would have been otherwise difficult to obtain in studying depressed individuals (Moberly \& Watkins, 2008) and persons with borderline personality disorder (Trull et al., 2008). Nevertheless, it is possible that there is some bias in participants' recall of affect and events. In the present study, for example, we obtained participants' reports of duration and intensity of physical activity since the last prompt. Importantly, there were no significant correlations between length of time interval between prompts and reported duration or intensity of physical activity, suggesting that recall of physical activity was equivalent for shorter and longer intervals between prompts and, therefore, likely not subject to recall bias. Future studies could address this issue more explicitly by adding accelerometers or heart rate monitors as a second, more objective measure for assessing physical activity. It is important to note, however, that such objective measures are not infallible in assessing activity. For example, accuracy of accelerometers is influenced by where on the body they are placed, and heart rate monitors are problematic in distinguishing increased heart rate attributable to activity from increased heart rate attributable to psychological states such as being nervous or excited.

The results of this study have a number of implications for our understanding and treatment of MDD. We found that self-initiated physical activity is significantly associated with PA in individuals diagnosed with MDD; indeed, even one bout of activity is associated with a higher level of PA over the day. Moreover, our findings indicate that exercise may act as a form of behavioral activation, a component of effective psychotherapies for depression (e.g., Brosse, Sheets, Lett, \& Blumenthal, 2002). Interestingly, Conn's (2010) meta-analysis documented a larger effect size for unsupervised than for supervised exercise in nonclinical samples, suggesting that supervised treatment interventions underestimate the effects of exercise on change in affect or depressive symptoms. Given the difficulty in motivating individuals to engage in exercise (e.g., Palomo et al., 2008), our findings illustrating the effects of even small amounts of self-initiated physical activity are particularly important. Therapists can work with their clients to integrate small units of physical activity into their daily schedules (e.g., taking the stairs instead of an elevator), which may be easier than trying to integrate bigger time-blocks of structured exercise. At the same time, it is important to note that we found a dose-response effect indicating that longer and more intense periods of activity lead to a greater increase in PA. Therefore, encouraging individuals with MDD to engage in more, especially more intense and more consistent, physical activity is also important (see also Haskell et al., 2007). For example, Silva et al. (2011) showed that increasing internal motivation for exercise through helping overweight sedentary women find activities that they enjoyed and about which they felt competent led to a sustained increase in physical activity over three years.

In sum, the present study demonstrates that self-initiated physical activity is significantly associated with an increase in PA, but not with a reduction in NA. It is noteworthy that the level of PA of individuals with MDD on prompts and days at which physical activity was reported was comparable to that of healthy control participants on prompts and days without physical activity, underscoring the importance and potential of integrating self-initiated physical activity into the treatment of individuals suffering from MDD.

\section{Acknowledgments}

The authors thank Patricia J. Deldin for her assistance in the study design, Sarah Victor for her help in conducting this study, as well as Krishna Savani, Susanne Scheibe, and Andrea H. Meyer for advice on the statistical analyses. 
This research was supported by Grants SFRH/BPD/ 35953/2007 from Fundação para a Ciência e a Tecnologia and Wi3496/4-1 from the Deutsche Forschungsgemeinschaft awarded to Jutta Mata, National Institute of Mental Health (NIMH) Grant Supplement MH74849 awarded to Renee J. Thompson, NIMH Grant MH60655 to John Jonides, and NIMH Grant MH59259 awarded to Ian H. Gotlib.

\section{References}

Abraham HD, Fava M. Order of onset of substance abuse and depression in a sample of depressed outpatients. Comprehensive Psychiatry. 1999; 40:44-50.10.1016/S0010-440X(99)90076-7 [PubMed: 9924877]

Arent SM, Landers DM, Etnier JL. The effects of exercise on mood in older adults: A meta-analytic review. Journal of Aging and Physical Activity. 2000; 8:407-430.

Bahrke MS, Morgan WP. Anxiety reduction following exercise and meditation. Cognitive Therapy and Research. 1978; 2:323-333.10.1007/BF01172650

Barrett, DJ.; Feldman Barrett, L. The Experience Sampling Program (ESP). 2000. Available at: http:// www.experience-sampling.org/

Bartholomew JB, Morrison D, Ciccolo JT. Effects of acute exercise on mood and well-being in patients with major depressive disorder. Medicine \& Science in Sports and Exercise. 2005; 37:2032-2037.10.1249/01.mss.0000178101.78322.dd [PubMed: 16331126]

Beck, AT.; Steer, RA.; Brown, GK. Manual for the Beck Depression Inventory-II. San Antonio, TX: Psychological Corporation; 1996.

Birnbaum HG, Kessler RC, Kelley D, Ben-Hamadi R, Joish VN, Greenberg PE. Employer burden of mild, moderate, and severe major depressive disorder: Mental health services utilization and costs, and work performance. Depression and Anxiety. 2010; 27:78-89.10.1002/Da.20580 [PubMed: 19569060]

Bodin T, Martinsen EW. Mood and self-efficacy during acute exercise in clinical depression. A randomized, controlled study. Journal of Sport \& Exercise Psychology. 2004; 26:623-633.

Brosse AL, Sheets ES, Lett HS, Blumenthal JA. Exercise and the treatment of clinical depression in adults - Recent findings and future directions. Sports Medicine. 2002; 32:741-760. [PubMed: 12238939]

Canli T, Sivers H, Thomason M, Whitfield S, Gabrieli JDE, Gotlib IH. Brain activation to emotional words in depressed versus healthy subjects. NeuroReport. 2004; 15:2585-2588. [PubMed: 15570157]

Clark MS, Milberg S, Ross J. Arousal cues arousal-related material in memory: Implications for understanding effects of mood on memory. Journal of Verbal Learning and Verbal Behavior. 1983; 22:633-649.10.1016/S0022-5371(83)90375-4

Cohen S, Doyle WJ, Turner RB, Alper CM, Skoner DP. Emotional style and susceptibility to the common cold. Psychosomatic Medicine. 2003; 65:652- 657.10.1097/01.PSY. 0000077508.57784.DA [PubMed: 12883117]

Conn VS. Depressive symptom outcomes of physical activity interventions: Meta-analysis findings. Annals of Behavioral Medicine. 2010; 39:128-138.10.1007/s12160-010-9172-x [PubMed: 20422333]

Cotman CW, Berchtold NC. Exercise: A behavioral intervention to enhance brain health and plasticity. Trends in Neurosciences. 2002; 25:295-301.10.1016/S0166-2236(02)02143-4 [PubMed: 12086747]

Craig CL, Marshall AL, Sjostrom M, Bauman AE, Booth ML, Ainsworth BE, Pekka O. International physical activity questionnaire: 12-country reliability and validity. Medicine and Science in Sports and Exercise. 2003; 35:1381-1395.10.1249/01.MSS.0000078924.61453.FB [PubMed: 12900694]

Deldin PJ, Deveney CM, Kim AS, Casas BR, Best JL. A slow wave investigation of working memory biases in mood disorders. Journal of Abnormal Psychology. 2001; 110:267281.10.1037/0021-843X.110.2.267 [PubMed: 11358021]

Dunn AL, Trivedi MH, O’Neal HA. Physical activity dose-response effects on outcomes of depression and anxiety. Medicine and Science in Sports and Exercise. 2001; 33:S587-S597. [PubMed: $11427783]$ 
Dunton GF, Atienza AA, Castro CM, King AC. Using ecological momentary assessment to examine antecedents and correlates of physical activity bouts in adults age 50+years: A pilot study. Annals of Behavioral Medicine. 2009; 38:249-255.10.1007/S12160-009-9141-4 [PubMed: 20052568]

Dunton GF, Whalen CK, Jamner LD, Henker B, Floro JN. Using ecologic momentary assessment to measure physical activity during adolescence. American Journal of Preventive Medicine. 2005; 29:281-287.10.1016/j.amepre.2005.07.020 [PubMed: 16242590]

Ebner-Priemer UW, Eid M, Kleindienst N, Stabenow S, Trull TJ. Analytic strategies for understanding affective (in)stability and other dynamic processes in psychopathology. Journal of Abnormal Psychology. 2009; 118(1):195-202.10.1037/A0014868 [PubMed: 19222325]

Eisenhower, D.; Mathiowetz, NA.; Morgenstein, D. Recall error: Sources and bias reduction techniques. In: Bieber, PP.; Groves, RM.; Lyberg, LE.; Mathiowetz, NA.; Sudman, S., editors. Measurement errors in surveys. New York: Wiley; 1991. p. 127-144.

Ekkekakis P. Pleasure and displeasure from the body: Perspectives from exercise. Cognition \& Emotion. 2003; 17:213-39.10.1080/02699930244000282

Ekman, P.; Friesen, WV.; Ellsworth, P. Emotion in the human face: Guidelines for research and an integration of findings. New York: Pergamon Press; 1972.

First, MB.; Spitzer, RL.; Gibbon, M.; Williams, JBW. The Structured Clinical Interview for DSM-IVTR Axis I disorders. New York: NY State Psychiatric Institute, Biometrics Research; 2001.

Focht BC, Gauvin L, Rejeski WJ. The contribution of daily experiences and acute exercise to fluctuations in daily feeling states among older, obese adults with knee osteoarthritis. Journal of Behavioral Medicine. 2004; 27:101-121.10.1023/B:JOBM.0000019847.80315.4d [PubMed: 15171102]

Gauvin L, Rejeski WJ, Norris JL. A naturalistic study of the impact of acute physical activity on feeling states and affect in women. Health Psychology. 1996; 15:391-397. [PubMed: 8891718]

Gauvin L, Rejeski WJ, Reboussin BA. Contributions of acute bouts of vigorous physical activity to explaining diurnal variations in feeling states in active, middle-aged women. Health Psychology. 2000; 19:365-375. [PubMed: 10907655]

George JM, Brief AP. Feeling good-doing good: A conceptual analysis of the mood at workorganizational spontaneity relationship. Psychological Bulletin. 1992; 112:310-329. [PubMed: 1454897]

Giacobbi PR, Hausenblas HA, Frye N. A naturalistic assessment of the relationship between personality, daily life events, leisure-time exercise, and mood. Psychology of Sport and Exercise. 2005; 6:67-81.10.1016/J.Psychsport.2004.10.009

Godin G, Shepard RJ. A simple method to assess exercise behavior in the community. Canadian Journal of Applied Sport Sciences. 1985; 10:141-146.

Gotlib IH, Joormann J. Cognition and depression: Current status and future directions. Annual Review of Clinical Psychology. 2010; 6:285-312.10.1146/annurev.clinpsy.121208.131305

Gotlib IH, Krasnoperova E, Yue DN, Joormann J. Attentional biases for negative interpersonal stimuli in clinical depression. Journal of Abnormal Psychology. 2004; 113:127-135.10.1037/0021-843x. 113.1.127

Grossman P, Deuring G, Garland SN, Campbell TS, Carlson TS. Patterns of objective physical functioning and perception of mood and fatigue in posttreatment breast cancer patients and healthy controls: An ambulatory psychophysiological investigation. Psychosomatic Medicine. 2008; 70:819-828.10.1097/PSY.0b013e31818106f1 [PubMed: 18725433]

Hamer M, Molloy GJ, de Oliveira C, Demakakos P. Leisure time physical activity, risk of depressive symptoms, and inflammatory mediators: The English Longitudinal Study of Ageing. Psychoneuroendocrinology. 2009; 34(7):1050-1055.10.1016/j.psyneuen.2009.02.004 [PubMed: 19272716]

Haskell WL, Lee IM, Pate RR, Powell KE, Blair SN, Franklin BA, Bauman A. Physical activity and public health: Updated recommendation for adults from the American College of Sports Medicine and the American Heart Association. Medicine and Science in Sports and Exercise. 2007; 39:1423-1434.10.1161/Circulationaha.107.185649 [PubMed: 17762377]

Jolly JB, Dyck MJ, Kramer TA, Wherry JN. Integration of positive and negative affectivity and cognitive content-specificity: Improved discrimination of anxious and depressive symptoms. 
Journal of Abnormal Psychology. 1994; 103:544-552.10.1037/0021-843X.103.3.544 [PubMed: 7930054]

Joormann J, Teachman BA, Gotlib IH. Sadder and less accurate? False memory for negative material in depression. Journal of Abnormal Psychology. 2009; 118:412-417.10.1037/a0015621 [PubMed: 19413415]

Kanning M, Schlicht W. Be active and become happy: An ecological momentary assessment of physical activity and mood. Journal of Sport \& Exercise Psychology. 2010; 32:253-261. [PubMed: 20479481]

Kessler, RC.; Wang, PS. The epidemiology of depression. In: Gotlib, IH.; Hammen, CL., editors. Handbook of depression. 2. New York: Guilford Press; 2009. p. 5-22.

Knapen J, Sommerijns E, Vancampfort D, Sienaert P, Pieters G, Haake P, Peuskens J. State anxiety and subjective well-being responses to acute bouts of aerobic exercise in patients with depressive and anxiety disorders. British Journal of Sports Medicine. 2009; 43:756-759.10.1136/bjsm. 2008.052654 [PubMed: 19019899]

Lawlor DA, Hopker SW. The effectiveness of exercise as an intervention in the management of depression: Systematic review and meta-regression analysis of randomised controlled trials. British Medical Journal. 2001; 322:763-767.10.1136/bmj.322.7289.763 [PubMed: 11282860]

Levens SM, Gotlib IH. Updating positive and negative stimuli in working memory in depression. Journal of Experimental Psychology: General. 2010; 139:654-664.10.1037/a0020283 [PubMed: 21038984]

Lindwall M, Rennemark M, Halling A, Berglund J, Hassmen P. Depression and exercise in elderly men and women: Findings from the Swedish National Study on Aging and Care. Journal of Aging and Physical Activity. 2007; 15(1):41-55. [PubMed: 17387228]

Mata J, Thompson RJ, Gotlib IH. BDNF genotype moderates the relation between physical activity and depressive symptoms. Health Psychology. 2010; 29(2):130-133.10.1037/a0017261 [PubMed: 20230085]

Mead GE, Morley W, Campbell P, Greig CA, McMurdo M, Lawlor DA. Exercise for depression. Cochrane Database of Systematic Reviews. 2009:Cd004366.10.1002/14651858.CD004366.pub4 [PubMed: 19588354]

Merikangas KR, Angst J, Eaton W, Canino G, Rubio-Stipec M, Wacker H, Kupfer DJ. Comorbidity and boundaries of affective disorders with anxiety disorders and substance misuse: Results of an international task force. British Journal of Psychiatry. 1996; 168(suppl 30):58-67.

Moberly NJ, Watkins ER. Ruminative self-focus and negative affect: An experience sampling study. Journal of Abnormal Psychology. 2008; 117:314-323.10.1037/0021-843X.117.2.314 [PubMed: 18489207]

Morgan WP. Psychogenic factors and exercise metabolism - a review. Medicine and Science in Sports and Exercise. 1985; 17:309-316. [PubMed: 3894867]

Nezlek JB. Multilevel random coefficient analyses of event- and interval-contingent data in social and personality psychology research. Personality and Social Psychology Bulletin. 2001; 27:771785.10.1177/0146167201277001

Palomo T, Beninger RJ, Kostrzewa RM, Archer T. Affective status in relation to impulsive, motor and motivational symptoms: Personality, development and physical exercise. Neurotoxicity Research. 2008; 14:151-168.10.1007/BF03033807 [PubMed: 19073423]

Phillips WJ, Hine DW, Thorsteinsson EB. Implicit cognition and depression: A meta-analysis. Clinical Psychology Review. 2010; 30:691-709.10.1016/j.cpr.2010.05.002 [PubMed: 20538393]

Pizzagalli DA, Iosifescu D, Hallett LA, Ratner KG, Fava M. Reduced hedonic capacity in major depressive disorder: Evidence from a probabilistic reward task. Journal of Psychiatric Research. 2008; 43:76-87.10.1016/j.jpsychires.2008.03.001 [PubMed: 18433774]

Reed J, Ones DS. The effect of acute aerobic exercise on positive activated affect: A meta-analysis. Psychology of Sport and Exercise. 2006; 7:477-514.10.1016/j.psychsport.2005.11.003

Silva MN, Markland D, Carraça EV, Vieira PN, Coutinho SR, Minderico CS, Teixeira PJ. Exercise autonomous motivation predicts 3-year weight loss in women. Medicine \& Science in Sports \& Exercise. 2011; 43:728-737. [PubMed: 20689448] 
Steer RA, Ball R, Ranieri WF, Beck AT. Dimensions of the Beck Depression Inventory-II in clinically depressed outpatients. Journal of Clinical Psychology. 1999; 55:117-128.10.1002/ (SICI)1097-4679(199901)55:1<117::AID-JCLP12>3.0.CO;2-A [PubMed: 10100838]

Sullivan LE, Fiellin DA, O'Connor PG. The prevalence and impact of alcohol problems in major depression: A systematic review. The American Journal of Medicine. 2005; 118:330-341.10.1016/ j.amjmed.2005.01.007 [PubMed: 15808128]

Thompson PD, Buchner D, Pina IL, Balady GJ, Williams MA, Marcus BH, Costa F. Exercise and physical activity in the prevention and treatment of atherosclerotic cardiovascular disease - A statement from the Council on Clinical Cardiology (Subcommittee on Exercise, Rehabilitation, and Prevention) and the Council on Nutrition, Physical Activity, and Metabolism (Subcommittee on Physical Activity). Circulation. 2003; 107(24):3109-3116.10.1161/01.ATV. 0000089628.63625.D4 [PubMed: 12821592]

Trull TJ, Solhan MB, Tragesser SL, Jahng S, Wood PK, Piasecki TM, Watson D. Affective instability: Measuring a core feature of borderline personality disorder with ecological momentary assessment. Journal of Abnormal Psychology. 2008; 117:647-661.10.1037/a0012532 [PubMed: 18729616]

Wadden TA, Stunkard AJ, Smoller JW. Dieting and depression: A methodological study. Journal of Consulting and Clinical Psychology. 1986; 54:869-871.10.1037/0022-006X.54.6.869 [PubMed: 3794038]

Watson D, Clark LA, Tellegen A. Development and validation of brief measures of positive and negative affect: The PANAS scales. Journal of Personality and Social Psychology. 1988; 54:10631070.10.1037/0022-3514.54.6.1063 [PubMed: 3397865]

Watson D, Clark LA, Weber K, Assenheimer JS. Testing a tripartite model: II. Exploring the symptom structure of anxiety and depression in student, adult, and patient samples. Journal of Abnormal Psychology. 1995; 104:15-25.10.1037/0021-843X.104.1.3 [PubMed: 7897037] 

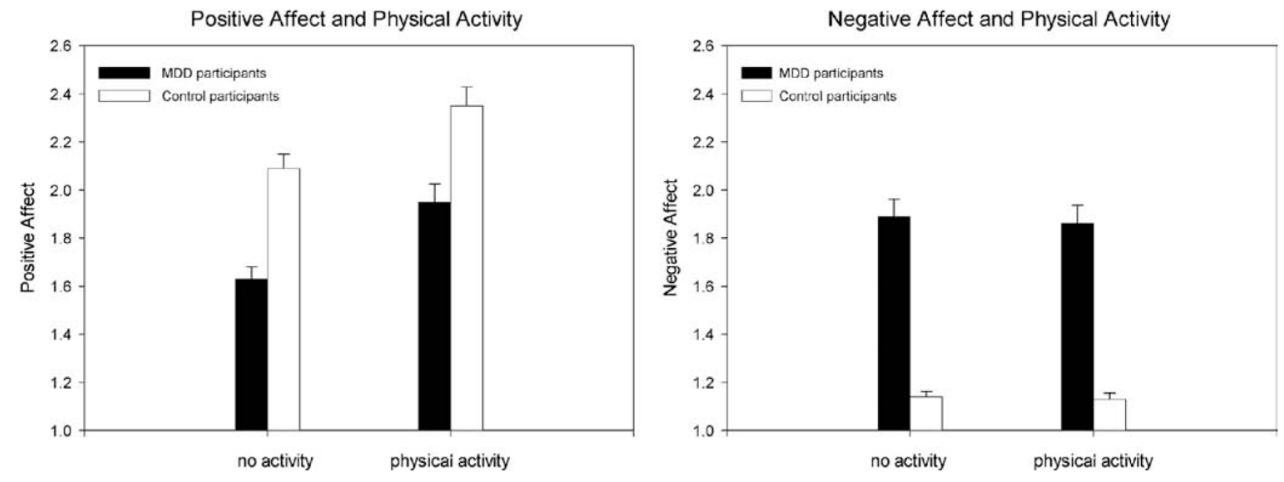

Figure 1.

Aggregated within-person affect (prompt level) separated by intensity level of activity and participant group. Error bars represent standard errors of the mean. Because HLM estimates the standard error of the difference between groups and not the standard errors of each mean value, the values shown in this graph are the aggregated mean of the intraindividual mean of each participant's affect over the experience sampling week; the standard errors are derived from this aggregated mean. The PA and NA values shown here are not adjusted for time-ofday effects. 

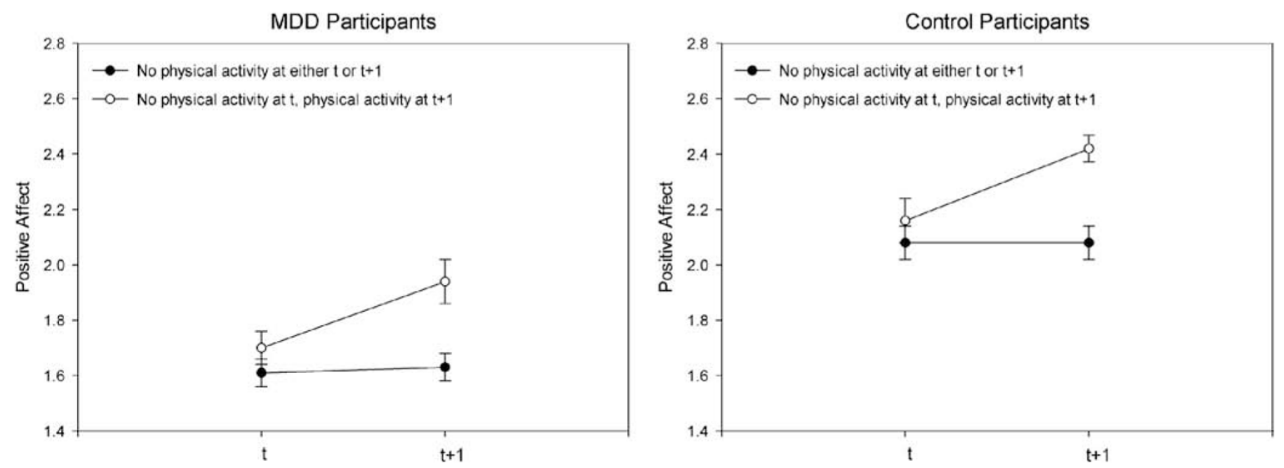

Figure 2.

PA over subsequent prompts at which physical activity either occurred at neither occasion or occurred between the first and second occasions, separated by participant group. Error bars represent standard errors of the mean. Because HLM estimates the standard error of the difference between groups and not the standard errors of each mean value, the values shown in this graph are the aggregated mean of the intraindividual mean of each participant's affect over the experience sampling week; the standard errors are derived from this aggregated mean. The PA values shown here are not adjusted for time-of-day effects. 
Table 1

Demographic Characteristics of Participants With MDD and Control Participants

\begin{tabular}{lccl}
\hline & MDD participants $(\boldsymbol{n}=\mathbf{5 3})$ & Control participants $(\boldsymbol{n}=\mathbf{5 3})$ & Statistical difference values \\
\hline BDI score, M (SD) & $32.2(8.6)$ & $1.7(2.3)$ & $t(104)=-24.86, p<.001$ \\
Age, M (SD) & $28.2(6.4)$ & $25.4(6.4)$ & $t(104)=-2.19, p=.03$ \\
Ethnicity $(n)$ & & $\chi^{2}(5)=7.79, p=.17$ \\
African American & 3 & 5 & \\
Asian & 2 & 9 & \\
Caucasian & 39 & 33 & \\
Hispanic & 2 & 1 & \\
Multiracial & 5 & 5 & \\
Other & 2 & 0 & \\
Education $(n)$ & & & \\
High school & 6 & 0 & \\
Some college & 20 & 25 & \\
Bachelor's & 23 & 23 & \\
Master's & 4 & 5 & \\
Gender $(n)$ & 38 & & \\
Female & 15 & 17 & \\
Male & & & \\
\hline
\end{tabular}

Note. MDD = Major Depressive Disorder; BDI = Beck Depression Inventory - II. 
Table 2

Descriptive Statistics For Within-Person Variables Over the Experience Sampling Week

\begin{tabular}{|c|c|c|c|}
\hline & MDD participants & Control participants & Statistical difference values \\
\hline Number of completed prompts, M (SD) & $44.0(7.6)$ & $42.4(7.8)$ & $t(104)=1.08, p=.29$ \\
\hline PA aggregated over the week, M (SD) & $1.7(0.4)$ & $2.2(0.5)$ & $t(104)=5.54, p<.001$ \\
\hline NA aggregated over the week, M (SD) & $1.9(0.5)$ & $1.1(0.2)$ & $t(104)=9.98, p<.001$ \\
\hline Times physically active during the week (median) & 6 & 7 & $U=1180.5, Z=-1.42, p=.16$ \\
\hline Times mildly active (median) & 3 & 2 & $U=1324.0, Z=-0.51, p=.61$ \\
\hline Times moderately active (median) & 2 & 2 & $U=1319.0, Z=-0.55, p=.58$ \\
\hline Times strenuously active (median) & 0 & 1 & $U=1319.0, Z=-2.09, p=.04$ \\
\hline METminutes (median) & 73 & 66 & $U=2624.5, Z=-0.69, p=.49$ \\
\hline Average duration of physical activity per bout in min, $M(S D)$ & $35.6(19.2)$ & $30.2(16.0)$ & $t(102)^{a}=-1.56, p=.12$ \\
\hline
\end{tabular}

Note . METminutes $=$ metabolic equivalent of task $\times$ minutes engaged in task.

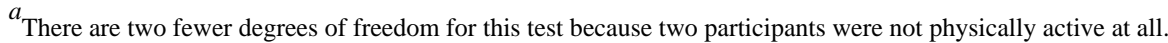

